\title{
Ruling Out the Massless Up-Quark Solution to the Strong $C P$ Problem by Computing the Topological Mass Contribution with Lattice QCD
}

\author{
Constantia Alexandrou $\odot,{ }^{1,2}$ Jacob Finkenrath $\odot,{ }^{2}$ Lena Funcke $\odot,{ }^{3}$ Karl Jansen, ${ }^{4}$ Bartosz Kostrzewa $\odot,{ }^{5,6}$ \\ Ferenc Pittler®, ${ }^{5,6}$ and Carsten Urbach $\oplus^{5,6}$ \\ ${ }^{1}$ Department of Physics, University of Cyprus, P.O. Box 20537, 1678 Nicosia, Cyprus \\ ${ }^{2}$ Computation-Based Science and Technology Research Center, \\ The Cyprus Institute, 20 Konstantinou Kavafi Street, 2121 Nicosia, Cyprus \\ ${ }^{3}$ Perimeter Institute for Theoretical Physics, 31 Caroline Street North, Waterloo, Ontario N2L 2Y5, Canada \\ ${ }^{4}$ NIC, DESY Zeuthen, Platanenallee 6, 15738 Zeuthen, Germany \\ ${ }^{5}$ Helmholtz Institut für Strahlen- und Kernphysik, University of Bonn, Nussallee 14-16, 53115 Bonn, Germany \\ ${ }^{6}$ Bethe Center for Theoretical Physics, University of Bonn, Nussallee 12, 53115 Bonn, Germany
}

(Received 13 March 2020; revised 20 July 2020; accepted 29 September 2020; published 2 December 2020)

\begin{abstract}
The infamous strong $C P$ problem in particle physics can in principle be solved by a massless up quark. In particular, it was hypothesized that topological effects could substantially contribute to the observed nonzero up-quark mass without reintroducing $C P$ violation. Alternatively to previous work using fits to chiral perturbation theory, in this Letter, we bound the strength of the topological mass contribution with direct lattice QCD simulations, by computing the dependence of the pion mass on the dynamical strangequark mass. We find that the size of the topological mass contribution is inconsistent with the massless upquark solution to the strong $C P$ problem.
\end{abstract}

DOI: 10.1103/PhysRevLett.125.232001

Introduction.-One of the unsolved puzzles in particle physics is the so-called strong $C P$ problem, where $C P$ stands for the combined charge conjugation and parity symmetry. In quantum chromodynamics (QCD), which is the theory of strong interactions, the nontrivial topological vacuum structure generates a $C P$-violating term

$$
\propto \theta G_{\mu \nu} \tilde{G}^{\mu \nu}
$$

in the Lagrangian, where $\theta$ is an a priori unknown parameter, $G$ is the gluon field strength tensor, and $\tilde{G}$ is its dual. However, experimentally, there is no sign of $C P$ violation in QCD. Instead, the strong upper bound $\theta \lesssim 10^{-10}[1-3]$ from measurements of the neutron electric dipole moment leads to a severe fine-tuning problem.

There are several proposals to overcome this problem, for instance, by postulating the existence of an axion [4-6]. A simple alternative could be the vanishing of the up-quark mass $m_{u}$, which at first sight seems inconsistent with results of current algebra. However, Refs. [7-10] pointed out that the up-quark mass in the chiral Lagrangian has two different contributions: a $C P$-violating perturbative

Published by the American Physical Society under the terms of the Creative Commons Attribution 4.0 International license. Further distribution of this work must maintain attribution to the author(s) and the published article's title, journal citation, and DOI. Funded by SCOAP ${ }^{3}$. contribution $m_{u}$ and a $C P$-conserving nonperturbative contribution $m_{\text {eff }}$ from topological effects, such as instantons. While $m_{u}=0$ could be easily ensured by an accidental symmetry [10-14], $m_{\text {eff }}$ does not contribute to the neutron electric dipole moment and is parametrically of order $m_{\mathrm{eff}} \sim m_{d} m_{s} / \Lambda_{\mathrm{QCD}}$, plausibly as large as the total required up-quark mass. Testing this simple solution to the strong $C P$ problem is particularly important because the other proposed solutions, including the axion [4-6] and Nelson-Barr $[15,16]$ mechanisms, face several theoretical challenges [17].

As the only tool to reliably test the $m_{u}=0$ proposal [10], lattice gauge theory has determined the up-quark mass to $m_{u}(2 \mathrm{GeV}) \sim 2 \mathrm{MeV}$ by fitting the light meson spectrum with errors around 5\% (see Ref. [18] for a review). As proposed in Refs. $[19,20]$, it would be beneficial to perform a complementary analysis by calculating the dependence of the pion mass on the dynamical strange-quark mass while keeping the light quark masses fixed. This direct calculation would have the advantage of avoiding any fitting procedures.

Lattice QCD simulations are now being performed, taking into account the first two quark generations as dynamical degrees of freedom. In addition, simulations are performed at (or very close to) the physical values of the pion, kaon, and $D$-meson masses [21]; and at various values of the lattice spacing and volumes, such that systematic effects can be studied and eventually controlled $[22,23]$. Finally, the theoretically sound definitions of the 
topological charge and susceptibility on the lattice (see Ref. [24] for a review) allow for directly accessing topological effects related to $m_{\text {eff }}$.

In this Letter, we perform a cross-check of the $m_{u}>0$ hypothesis based on the proposals of Refs. $[19,20]$. In particular, we compute the parameter $\beta_{2} / \beta_{1}$, which measures the strength of $m_{\mathrm{eff}}$ and probes the contribution of small instantons and other topological effects to the chiral Lagrangian. While $\beta_{2} / \beta_{1}$ is usually obtained from a combination of low-energy constants [25], this indirect lattice method requires chiral perturbation theory $(\chi \mathrm{PT})$. Using direct lattice computations instead, we obtain the result $\beta_{2} / \beta_{1}=0.63(39) \mathrm{GeV}^{-1}$ by computing the dependence of the pion mass on the strange-quark mass. Since a bound significantly smaller than $5 \mathrm{GeV}^{-1}$ provides an exclusion of the massless up-quark hypothesis [10,20], our result rules out this hypothesis, in accordance with previous fits of $\chi \mathrm{PT}$ to lattice data [18,25-27].

Method.-We test the $m_{u}=0$ proposal by investigating the variation of the pion mass with respect to the strangequark mass. The general form of the quark-mass dependence of the pion mass reads [28]

$M_{\pi}^{2}=\beta_{1}\left(m_{u}+m_{d}\right)+\beta_{2} m_{s}\left(m_{u}+m_{d}\right)+$ higher orders,

where the first term is the first-order contribution of the light quark masses in $\chi \mathrm{PT}$. The second term receives contributions both from small instantons that could mimic a nonzero $m_{u}$ and from higher-order terms in $\chi \mathrm{PT}$ that are proportional to $m_{s}$, including logarithmic corrections. In order to let topological effects explain the observed value for $m_{u}$ and to allow for a solution of the strong $C P$ problem, $\beta_{2} / \beta_{1} \approx 5 \mathrm{GeV}^{-1}$ at renormalization scale $\bar{\mu}=2 \mathrm{GeV}$ in the modified minimal subtraction scheme ( $\overline{\mathrm{MS}}$ scheme) is required [20].

The most precise and computationally challenging test of the ratio $\beta_{2} / \beta_{1}$ is to vary either the strange-quark mass or the light quark mass, $m_{u}=m_{d} \equiv m_{\ell}$. For example, by varying $m_{s}$ while keeping $m_{\ell}$ fixed, we obtain $[19,20]$

$$
\frac{\beta_{2}}{\beta_{1}} \approx \frac{M_{\pi, 1}^{2}-M_{\pi, 2}^{2}}{m_{s, 1} M_{\pi, 2}^{2}-m_{s, 2} M_{\pi, 1}^{2}},
$$

where $M_{\pi, i}=M_{\pi}\left(m_{s, i}\right)$ is the average pion mass as a function of the varied strange-quark mass $m_{s, i}$ at fixed $m_{\ell}$. Note that the approximate result for $\beta_{2} / \beta_{1}$ in Eq. (2) is independent of the up and down quark masses. Crucially, this allows us to reliably compute $\beta_{2} / \beta_{1}$ even at larger than physical quark masses. The higher-order corrections in Eq. (1) reintroduce a small residual pion-mass dependence for $\beta_{2} / \beta_{1}$ that finally needs to be cancelled by a chiral extrapolation.

While this challenging direct method to compute the ratio $\beta_{2} / \beta_{1}$ is independent of $\chi \mathrm{PT}$, the more common indirect method is to use the chiral Lagrangian. For example, Ref. [20] used lattice data from the Flavour Lattice Averaging Group report of 2013 [29] to estimate $\beta_{2} / \beta_{1} \simeq(1 \pm 1) \mathrm{GeV}^{-1}$, neglecting chiral logarithms and higher-order terms in the chiral Lagrangian. To check the consistency of our computations with the results of Ref. [20], we have also computed $\beta_{2} / \beta_{1}$ indirectly by using chiral fits and measuring $M_{K}^{2}\left(m_{s}\right)$, obtaining excellent agreement with Ref. [20].

Lattice computation.-In this Letter, we use gauge configurations generated by the Extended Twisted Mass Collaboration with the Iwasaki gauge action [30] and Wilson twisted mass fermions at maximal twist [31,32] with up, down, strange, and charm dynamical quark flavors. Up and down quarks are mass degenerate. All the gauge configuration ensembles we used are listed together with the corresponding pion- and strange-quarkmass values in Table I. For details on how these values are obtained, we refer to the Supplemental Material [33].

We first perform the analysis using three sets each with a pair of ensembles ( $A X$ and $A X s$ with $X=60,80$, or 100) without the so-called clover term in the action. Details on the production of these ensembles can be found in Ref. [42]. Each pair with $X=60,80$, and 100 has identical parameters apart from strange- and charm quark-mass values, which are close to their physical values. The three pairs have equal strange- and charm quark masses within errors but differ in the light quark-mass value corresponding to unphysically large pion-mass values of about 386, 444 , and $494 \mathrm{MeV}$, respectively. The lattice spacing value corresponds to $a=0.0885(36) \mathrm{fm}$ [34] determined from the pion decay constant $f_{\pi}$.

In addition, we use one ensemble ( $c A 211.30 .32)$ that includes the clover term in the action [23]. While Wilson twisted mass fermions at maximal twist automatically remove discretization effects linear in the lattice spacing $a$ [43], and thus leave only lattice artifacts at $\mathcal{O}\left(a^{2}\right)$, the clover term reduces these $\mathcal{O}\left(a^{2}\right)$ effects even further [44]. The $c A 211.30 .32$ ensemble has a smaller pion-mass value of about $270 \mathrm{MeV}$ as well as strange- and charm

TABLE I. Pion- and strange-quark masses in physical units for the ensembles used in this work. The strange-quark mass is quoted at $2 \mathrm{GeV}$ in the $\overline{\mathrm{MS}}$ scheme.

\begin{tabular}{lcc}
\hline \hline Ensemble & $M_{\pi}[\mathrm{MeV}]$ & $m_{s}[\mathrm{MeV}]$ \\
\hline$A 60$ & $386(16)$ & $98(4)$ \\
$A 60 s$ & $387(16)$ & $79(4)$ \\
$A 80$ & $444(18)$ & $98(4)$ \\
$A 80 s$ & $443(18)$ & $79(4)$ \\
$A 100$ & $494(20)$ & $100(4)$ \\
$A 100 s$ & $495(20)$ & $79(4)$ \\
$c A 211.30 .32$ & $276(3)$ & $99(2)$ \\
$c A 211.30 .32 l$ & $275(3)$ & $94(2)$ \\
$c A 211.30 .32 h$ & $276(3)$ & $104(2)$ \\
\hline \hline
\end{tabular}


quark-mass values again close to their physical values. The lattice spacing value is $a=0.0896(10) \mathrm{fm}$, determined using the nucleon mass dependence on the pion mass. This estimation is done by employing $\chi \mathrm{PT}$ at $\mathcal{O}\left(p^{3}\right)[45,46]$, where $p$ is a typical meson momentum. Similarly to Ref. [23], the $N_{f}=2+1+1$ nonclover twisted mass ensembles [47] at different lattice spacings, which also include the $A X$ ensembles, were used to control the chiral extrapolations.

Since the pion mass of the $c A 211.30 .32$ ensemble is significantly smaller than the ones of the $A X$ ensembles, and thus closer to the physical value, we consider this ensemble as the most appropriate one to compute our final value of $\beta_{2} / \beta_{1}$. Moreover, $c A 211.30 .32$ uses the same action as ensembles that are currently under production with a physical value of the pion mass. These ensembles could be used, in principle, in future work to repeat the calculation presented here at the physical point. For $c A 211.30 .32$, we have simulations for only one dynamical strange-quark mass; thus, it is necessary to apply the socalled reweighting technique to investigate the strangequark-mass dependence of $M_{\pi}$ while keeping the charm and light quark masses constant [33]. We denote with $c A 211.30 .32 l(c A 211.30 .32 h)$ the reweighted ensemble with a 5\% lower (higher) strange-quark-mass value than the original ensemble $c A 211.30 .32$.

In contrast, for the $A X(\mathrm{~s})$ ensembles, we have pairs of ensembles with different dynamical strange-quark masses; thus, we can use a direct approach to investigate the strange-quark-mass dependence of $M_{\pi}$. Note that in this case also, the charm quark mass differs slightly, but its value is so close to the cutoff that this difference will not affect our results. While the $A X(s)$ ensembles have rather heavy pion masses (see Table I), they are ideal to test the robustness of the reweighting procedure that we apply to $c A 211.30 .32$. In fact, we use these ensembles to demonstrate that reweighting works successfully [33]. In addition, the $\beta_{2} / \beta_{1}$ values from these ensembles provide an insight into the pion-mass dependence of $\beta_{2} / \beta_{1}$.

Results.-Using the values of $M_{\pi}$ and $m_{s}$ from Table I as input (more precisely, the corresponding values in lattice units [33]), we compute $\beta_{2} / \beta_{1}$ from Eq. (2). The results from this direct approach for the three pairs $A 60(s), A 80(s)$ and $A 100(s)$ are compiled in Table II, where we quote $\beta_{2} / \beta_{1}$, as well as $\beta_{1}$ and $\beta_{2}$ separately. Since the pion-mass differences are all zero within errors (see Table I), we find that $\beta_{2} / \beta_{1}$ is compatible with zero as well. Note that the errors of the observables compiled in Table I are correlated per ensemble. This correlation is taken into account in our analysis for $\beta_{2} / \beta_{1}$.

Finally, we use reweighting on the $c A 211.30 .32$ ensemble to vary the strange-quark mass by $\pm 5 \%$ around its original value. The change in the pion mass with the strange-quark mass is not significant; see Table I. The corresponding values for $\beta_{2} / \beta_{1}$ [33] are again compiled in
TABLE II. Results for $\beta_{2}, \beta_{1}$, and $\beta_{2} / \beta_{1}$ from Eq. (2) in physical units for all ensembles at $\bar{\mu}=2 \mathrm{GeV}$ in the $\overline{\mathrm{MS}}$ scheme.

\begin{tabular}{lrlr}
\hline \hline Ensemble & $\beta_{2}\left[\mathrm{GeV}^{2}\right]$ & $\beta_{1}\left[\mathrm{GeV}^{3}\right]$ & $\beta_{2} / \beta_{1}\left[\mathrm{GeV}^{-1}\right]$ \\
\hline$A 60(s)$ & $-0.0009(08)$ & $0.0029(4)$ & $-0.32(26)$ \\
$A 80(s)$ & $0.0005(10)$ & $0.0036(4)$ & $0.15(30)$ \\
$A 100(s)$ & $-0.0010(10)$ & $0.0053(6)$ & $-0.19(19)$ \\
$c A 211.30 .32(h)$ & $0.00007(11)$ & $0.00039(5)$ & $0.18(30)$ \\
$c A 211.30 .32(l)$ & $0.00026(11)$ & $0.00037(5)$ & $0.69(33)$ \\
$c A 211.30 .32(h, l)$ & $0.00033(12)$ & $0.00076(5)$ & $0.43(16)$ \\
\hline \hline
\end{tabular}

Table II. Here, we denote with $c A 211.30 .32(h)$ the value for $\beta_{2} / \beta_{1}$ obtained from the combination of the ensembles $c A 211.30 .32 h$ and $c A 211.30 .32$. Likewise, $c A 211.30 .32(l)$ is the combination of $c A 211.30 .32$ and $c A 211.30 .32 l$, while $c A 211.30 .32(h, l)$ is the combination of $c A 211.30 .32 h$ and $c A 211.30 .32 l$.

In Fig. 1 , we show the values of the ratio $\beta_{2} / \beta_{1}$ at $\bar{\mu}=$ $2 \mathrm{GeV}$ in the $\overline{\mathrm{MS}}$ scheme as a function of the squared pion mass $M_{\pi}^{2}$ in physical units. The three blue points at heavier pion-mass values correspond to the three pairs of the $A X(s)$ ensembles without the clover term. The three red points at lower pion-mass values correspond to the $c A 211.30 .32$ ensemble including the clover term. The latter three points are slightly displaced horizontally for better legibility. While all of the points are compatible with zero at the $1.5 \sigma$ level, we observe a slight trend toward larger $\beta_{2} / \beta_{1}$ values with decreasing pion-mass values.

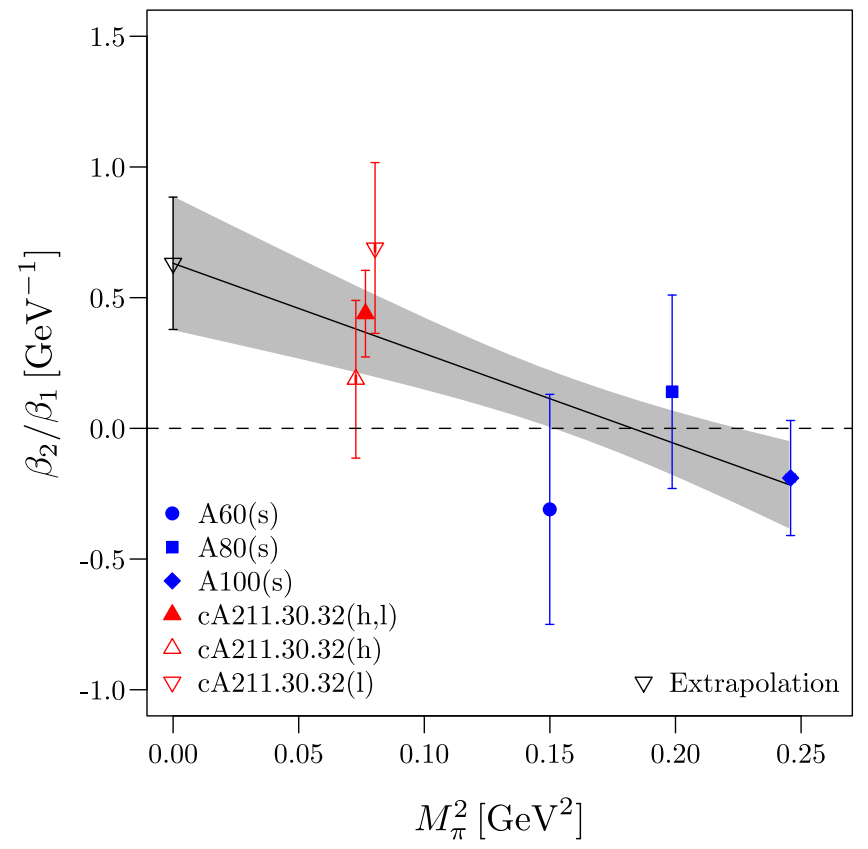

FIG. 1. The ratio $\beta_{2} / \beta_{1}$ as a function of the squared pion mass $M_{\pi}^{2}$ in physical units. The solid line with the $1 \sigma$ error band represents a linear extrapolation in $M_{\pi}^{2}$. We extrapolate to the chiral limit to eliminate higher-order corrections to $\beta_{2} / \beta_{1}$; see Eqs. (1) and (2). 
In addition, we show in Fig. 1 a linear extrapolation of $\beta_{2} / \beta_{1}$ in $M_{\pi}^{2}$ to the chiral limit. This linear dependence can be justified with $\chi \mathrm{PT}$, which predicts [18]

$$
\frac{\beta_{2}}{\beta_{1}} \approx \frac{\alpha_{2}}{\alpha_{1}+\left(\alpha_{3} / \alpha_{1}\right) M_{\pi}^{2}} \approx \frac{\alpha_{2}}{\alpha_{1}}-\frac{\alpha_{2} \alpha_{3}}{\alpha_{1}^{3}} M_{\pi}^{2}
$$

modulo logarithmic corrections, where $\alpha_{1,2,3}$ are combinations of low-energy constants with $\alpha_{1} \gg\left(\alpha_{3} / \alpha_{1}\right) M_{\pi}^{2}$, and $M_{\pi}^{2}=\alpha_{1} m_{\ell}+\mathcal{O}\left(\alpha_{2,3}\right)$ with $\mathcal{O}\left(\alpha_{2,3}\right) /\left(\alpha_{1} m_{\ell}\right) \approx 0.1$. Since the data points for ensemble $c A 211.30 .32$ are highly correlated, we include only the combination of $c A 211.30 .32 h$ and $c A 211.30 .32 l$ in the fit denoted as $c A 211.30 .32(h, l)$ in Table II. The fit has $\chi^{2} /$ d.o.f. $=$ $3.28 / 2$ (i.e., a $p$ value of 0.2 ) and the chirally extrapolated value reads $\beta_{2} / \beta_{1}=0.63(25) \mathrm{GeV}^{-1}$. As mentioned, we extrapolate to the chiral limit to cancel the residual pionmass dependence in Eq. (3), which stems from higher-order corrections in Eq. (1) and does not appear in the expression for $\beta_{2} / \beta_{1}$ in Eq. (2). Our data thus confirm in hindsight that the approximation in Eq. (2) is justified.

Discussion.-All the estimates for the ratio $\beta_{2} / \beta_{1}$ presented in this Letter are consistent with zero at the $1.5 \sigma$ level. With the chiral extrapolation explained above and $1 \sigma$ statistical uncertainty, we exclude a value of $5 \mathrm{GeV}^{-1}$ by an amount significantly larger than $10 \sigma$. The remaining question is whether there are additional systematic uncertainties that could potentially spoil this conclusion.

Let us first consider the discretization errors for $\beta_{2} / \beta_{1}$, which are of order $\left(a \Lambda_{\mathrm{QCD}}\right)^{2}$ multiplied by an unknown coefficient, with $\Lambda_{\mathrm{QCD}}=341(12) \mathrm{MeV}$ [48]. We can reliably estimate the coefficient by using the known continuum extrapolation values for $M_{\pi}^{2}$ and $m_{s}$ for the $A X$ ensembles [34]. By comparing these continuum values to our lattice results for $M_{\pi}^{2}$ and $m_{s}$, we can infer the size of discretization errors at our given lattice spacing. Depending on the scaling variable, the discretization errors in $M_{\pi}^{2}$ and the strange-quark mass are both on the order of $5 \%-10 \%$. If propagated generously, this implies a $10 \%$ uncertainty on the numerator, a $15 \%$ uncertainty on the denominator, and thus about $20 \%$ on the ratio $\beta_{2} / \beta_{1}$. Note that this estimate is highly conservative because most of the discretization effects cancel in the differences in both the numerator and the denominator. Because of the reduced lattice artifacts with the action including the clover term (see Supplemental Material [33]), we do not expect larger uncertainties on the ratio for the ensemble $c A 211.30 .32$ stemming from discretization effects.

In addition, there is a residual pion-mass dependence of $\beta_{2} / \beta_{1}$, which we account for by extrapolating to the chiral limit. In this extrapolation, the errors stemming from different lattice artifacts of the $A X$ and $c A 211.30 .32$ ensembles are taken into account by the above-mentioned $20 \%$ uncertainty. Last, there are finite-size effects for $M_{\pi}$ proportional to $\exp \left(-M_{\pi} L\right)$, with $L$ as the spatial extent of the lattice, but no finite-size corrections to $m_{s}$. Since the strange-quark-mass dependence of $M_{\pi}$ is so weak, these finite-size effects are equal for $M_{\pi, 1}^{2}$ and $M_{\pi, 2}^{2}$, and thus cancel in the ratio $\beta_{2} / \beta_{1}$.

In summary, taking the chirally extrapolated value for $\beta_{2} / \beta_{1}$ plus the $1 \sigma$ statistical error and the $20 \%$ uncertainty for discretization effects, we arrive at the following conservative estimate:

$$
\begin{aligned}
\frac{\beta_{2}}{\beta_{1}} & =0.63(25)_{\text {stat }}(14)_{\text {sys }} \mathrm{GeV}^{-1} \\
& =0.63(39) \mathrm{GeV}^{-1}
\end{aligned}
$$

at $\bar{\mu}=2 \mathrm{GeV}$ in the $\overline{\mathrm{MS}}$ scheme. For the final estimate, we have added the errors linearly. Note that our data are equally well compatible with a constant extrapolation in $M_{\pi}^{2}$, which would lead to a significantly smaller value at the physical point. Thus, we consider Eq. (4) as a conservative estimate. Moreover, the logarithmic corrections from chiral perturbation theory contributing to $\beta_{2} / \beta_{1}$ (see, e.g., Refs. $[49,50])$ are of the same order as our value in Eq. (4); therefore, the topological contribution to $\beta_{2} / \beta_{1}$ should be even smaller.

Conclusion.- In this Letter, we have tested the massless up-quark solution to the strong $C P$ problem by directly investigating the strange-quark-mass dependence of the pion mass on the lattice. This allows us to determine the ratio $\beta_{2} / \beta_{1}$, which would need to be larger than $5 \mathrm{GeV}^{-1}$ to solve the strong $C P$ problem.

Since all our estimates of $\beta_{2} / \beta_{1}$ are compatible with zero, we obtain a strong upper bound for $\beta_{2} / \beta_{1}$ including residual uncertainties stemming from discretization errors and chiral extrapolation. The result in Eq. (4) is clearly incompatible with the massless up-quark solution to the strong $C P$ problem. This exclusion of the $m_{u}=0$ solution is consistent with previous results using $\chi \mathrm{PT}$ and direct fits of the light meson spectrum.

Given our conservative error estimates, we consider it highly unlikely that the factor of 5 needed to rescue the solution to the strong $C P$ problem is hidden in the quoted uncertainties. A confirmation of this result using ensembles with physical pion-mass values could be undertaken in the future, once different values for the lattice spacing become available for a continuum extrapolation.

Our direct lattice results also quantitatively support the large- $N$ picture as a good description of QCD at low scales because the coefficient of the nonperturbatively induced mass operator is known to be suppressed in the large- $N$ limit $[10,19]$. Thus, our computations reliably demonstrate that the topological vacuum contributions to the chiral Lagrangian are negligible.

We thank all members of the ETM Collaboration for the most enjoyable collaboration. We also thank J. Gasser, D. Kaplan, T. Banks, Y. Nir, and N. Seiberg for helpful 
comments on the draft; and U.-G. Meißner for useful comments and discussions. We kindly thank F. Manigrasso and $\mathrm{K}$. Hadjiyiannakou for providing the necessary correlators for computing the nucleon mass on ensemble $c A 211.30 .32$. The authors gratefully acknowledge the Gauss Centre for Supercomputing e.V. [51] for funding this project by providing computing time on the GCS Supercomputer JUQUEEN [52] and the John von Neumann Institute for Computing (NIC) for computing time provided on the supercomputer JURECA [53] and JUWELS at Jülich Supercomputing Centre (JSC) under the projects hch02, ecy00, and hbn28. The project used resources of the SuperMUC at Leibniz Supercomputing Centre under the Gauss Centre for Supercomputing e.V. project pr74yo. The $c A 211.30 .32$ ensemble was generated on the Marconi-KNL supercomputer at CINECA within PRACE project Pra13-3304. This project was funded in part by the DFG as a project in the Sino-German CRC110 (TRR110) and by the PRACE Fifth and Sixth Implementation Phase (PRACE-5IP and PRACE-6IP) program of the European Commission under Grant Agreements No. 730913 and No. 823767. Research at Perimeter Institute is supported in part by the Government of Canada through the Department of Innovation, Science and Industry Canada and by the Province of Ontario through the Ministry of Colleges and Universities. The open source software packages tmLQCD [54], LEMON [55], DDalphaAMG [56-58], QUDA [59-61], and R [62] have been used.

[1] R. J. Crewther, P. Di Vecchia, G. Veneziano, and E. Witten, Chiral estimate of the electric dipole moment of the neutron in quantum chromodynamics, Phys. Lett. B 88, 123 (1979).

[2] J. M. Pendlebury, S. Afach, N. J. Ayres, C. A. Baker, G. Ban et al., Revised experimental upper limit on the electric dipole moment of the neutron, Phys. Rev. D 92, 092003 (2015).

[3] C. Abel et al., Measurement of the Permanent Electric Dipole Moment of the Neutron, Phys. Rev. Lett. 124, 081803 (2020).

[4] R. D. Peccei and H. R. Quinn, CP Conservation in the Presence of Pseudoparticles, Phys. Rev. Lett. 38, 1440 (1977).

[5] S. Weinberg, A New Light Boson?, Phys. Rev. Lett. 40, 223 (1978).

[6] F. Wilczek, Problem of Strong $P$ and $T$ Invariance in the Presence of Instantons, Phys. Rev. Lett. 40, 279 (1978).

[7] H. Georgi and I. N. McArthur, Instantons and the u quark mass, Harvard preprint Report No. HUTP-81/A011, 1981.

[8] D. B. Kaplan and A. V. Manohar, Current-Mass Ratios of the Light Quarks, Phys. Rev. Lett. 56, 2004 (1986).

[9] K. Choi, C. W. Kim, and W. K. Sze, Mass Renormalization by Instantons and the Strong CP Problem, Phys. Rev. Lett. 61, 794 (1988).

[10] T. Banks, Y. Nir, and N. Seiberg, Missing (up) mass, accidental anomalous symmetries, and the strong $C P$ problem, in Yukawa Couplings and the Origins of Mass. Proceedings, 2nd IFT Workshop, Gainesville, USA (1994), pp. 26-41 [arXiv:hep-ph/9403203].

[11] M. Leurer, Y. Nir, and N. Seiberg, Mass matrix models, Nucl. Phys. B398, 319 (1993).

[12] M. Leurer, Y. Nir, and N. Seiberg, Mass matrix models: The sequel, Nucl. Phys. B420, 468 (1994).

[13] A. E. Nelson and M. J. Strassler, A realistic supersymmetric model with composite quarks, Phys. Rev. D 56, 4226 (1997).

[14] D. E. Kaplan, F. Lepeintre, A. Masiero, A. E. Nelson, and A. Riotto, Fermion masses and gauge mediated supersymmetry breaking from a single $U(1)$, Phys. Rev. D 60, 055003 (1999).

[15] A. E. Nelson, Naturally weak $C P$ violation, Phys. Lett. 136B, 387 (1984).

[16] S. M. Barr, Solving the Strong CP Problem without the Peccei-Quinn Symmetry, Phys. Rev. Lett. 53, 329 (1984).

[17] M. Dine and P. Draper, Challenges for the Nelson-Barr mechanism, J. High Energy Phys. 08 (2015) 132.

[18] S. Aoki et al. (Flavour Lattice Averaging Group), FLAG review 2019, Eur. Phys. J. C 80, 113 (2020).

[19] A. G. Cohen, D. B. Kaplan, and A.E. Nelson, Testing $m(u)=0$ on the lattice, J. High Energy Phys. 11 (1999) 027.

[20] M. Dine, P. Draper, and G. Festuccia, Instanton effects in three flavor QCD, Phys. Rev. D 92, 054004 (2015).

[21] Note that, in this work, we can use larger than physical quark masses due to reasons discussed below Eq. (2).

[22] A. Bazavov et al. (MILC Collaboration), Lattice QCD ensembles with four flavors of highly improved staggered quarks, Phys. Rev. D 87, 054505 (2013).

[23] C. Alexandrou et al., Simulating twisted mass fermions at physical light, strange and charm quark masses, Phys. Rev. D 98, 054518 (2018).

[24] C. Alexandrou, A. Athenodorou, K. Cichy, A. Dromard, E. Garcia-Ramos, K. Jansen, U. Wenger, and F. Zimmermann, Comparison of topological charge definitions in lattice QCD, Eur. Phys. J. C 80, 424 (2020).

[25] D. R. Nelson, G. T. Fleming, and G. W. Kilcup, Is Strong $C P$ due to a Massless Up Quark?, Phys. Rev. Lett. 90, 021601 (2003).

[26] J. M. Cline, Can $\theta_{\mathrm{QCD}}=\pi$ ?, Phys. Rev. Lett. 63, 1338 (1989).

[27] J. Dragos, T. Luu, A. Shindler, J. de Vries, and A. Yousif, Confirming the existence of the strong $C P$ problem in lattice QCD with the gradient flow, arXiv:1902.03254 [Phys. Rev. $\mathrm{C}$ (to be published)].

[28] Note that $\beta_{1}$ is dimensionless as in Ref. [10], while the dimensionful $\beta_{2}$ translates to $\beta_{2} / \Lambda_{\chi \mathrm{SB}}$ in Ref. [10].

[29] S. Aoki et al., Review of lattice results concerning lowenergy particle physics, Eur. Phys. J. C 74, 2890 (2014).

[30] Y. Iwasaki, Renormalization group analysis of lattice theories and improved lattice action: Two-dimensional nonlinear $\mathrm{O}(N)$ sigma model, Nucl. Phys. B258, 141 (1985).

[31] R. Frezzotti, P. A. Grassi, S. Sint, and P. Weisz (Alpha Collaboration), Lattice QCD with a chirally twisted mass term, J. High Energy Phys. 08 (2001) 058. 
[32] R. Frezzotti and G. C. Rossi, Twisted mass lattice QCD with mass nondegenerate quarks, Nucl. Phys. B, Proc. Suppl. 128, 193 (2004).

[33] See Supplemental Material at http://link.aps.org/ supplemental/10.1103/PhysRevLett.125.232001 for strange-quark reweighting toward maximal twist, which includes Refs. [23,34-41].

[34] N. Carrasco et al. (European Twisted Mass Collaboration), Up, down, strange and charm quark masses with $N_{f}=$ $2+1+1$ twisted mass lattice QCD, Nucl. Phys. B887, 19 (2014).

[35] B. Sheikholeslami and R. Wohlert, Improved continuum limit lattice action for QCD with Wilson fermions, Nucl. Phys. B259, 572 (1985).

[36] R. Frezzotti and G. C. Rossi, Chirally improving Wilson fermions. II. Four-quark operators, J. High Energy Phys. 10 (2004) 070.

[37] K. Ottnad and C. Urbach (ETM Collaboration), Flavorsinglet meson decay constants from $N_{f}=2+1+1$ twisted mass lattice QCD, Phys. Rev. D 97, 054508 (2018).

[38] A. Hasenfratz, R. Hoffmann, and S. Schaefer, Reweighting toward the chiral limit, Phys. Rev. D 78, 014515 (2008).

[39] J. Finkenrath, F. Knechtli, and B. Leder, One flavor mass reweighting in lattice QCD, Nucl. Phys. B877, 441 (2013); Erratum, Nucl. Phys. 880, 574 (2014).

[40] B. Leder and J. Finkenrath, Tuning of the strange quark mass with optimal reweighting, Proc. Sci., LATTICE2014 (2015) 040 [arXiv:1501.06617].

[41] J. Finkenrath, F. Knechtli, and B. Leder, Isospin effects by mass reweighting, Proc. Sci., LATTICE2014 (2015) 297 [arXiv:1501.06441].

[42] R. Baron et al., Light hadrons from lattice QCD with light $(u, d)$, strange and charm dynamical quarks, J. High Energy Phys. 06 (2010) 111.

[43] R. Frezzotti and G. C. Rossi, Chirally improving Wilson fermions. I: $O(a)$ improvement, J. High Energy Phys. 08 (2004) 007.

[44] A. Abdel-Rehim, C. Alexandrou, F. Burger, M. Constantinou, P. Dimopoulos et al. (ETM Collaboration), First physics results at the physical pion mass from $N_{f}=2$ Wilson twisted mass fermions at maximal twist, Phys. Rev. D 95, 094515 (2017).

[45] J. Gasser, M. E. Sainio, and A. Svarc, Nucleons with chiral loops, Nucl. Phys. B307, 779 (1988).

[46] B. C. Tiburzi and A. Walker-Loud, Hyperons in two flavor chiral perturbation theory, Phys. Lett. B 669, 246 (2008).

[47] C. Alexandrou, V. Drach, K. Jansen, C. Kallidonis, and G. Koutsou, Baryon spectrum with $N_{f}=2+1+1$ twisted mass fermions, Phys. Rev. D 90, 074501 (2014).

[48] M. Bruno, M. D. Brida, P. Fritzsch, T. Korzec, A. Ramos, S. Schaefer, H. Simma, S. Sint, and R. Sommer (ALPHA Collaboration), QCD Coupling from a Nonperturbative
Determination of the Three-Flavor $\Lambda$ Parameter, Phys. Rev. Lett. 119, 102001 (2017).

[49] V. A. Novikov, M. A. Shifman, A. I. Vainshtein, and V. I. Zakharov, Are all hadrons alike?, Nucl. Phys. B191, 301 (1981).

[50] J. Gasser and H. Leutwyler, Chiral perturbation theory: Expansions in the mass of the strange quark, Nucl. Phys. B250, 465 (1985).

[51] www.gauss-centre.eu.

[52] M. Stephan and J. Docter, Jülich Supercomputing Centre, JUQUEEN: IBM blue gene/Q supercomputer system at the Jülich Supercomputing Centre, J. Large-Scale Res. Facil. 1, A1 (2015).

[53] D. Krause and P. Thörnig, Jülich Supercomputing Centre, JURECA: Modular supercomputer at Jülich Supercomputing Centre, J. Large-Scale Res. Facil. 4, A132 (2018).

[54] K. Jansen and C. Urbach, tmLQCD: A program suite to simulate Wilson twisted mass lattice QCD, Comput. Phys. Commun. 180, 2717 (2009).

[55] A. Deuzeman, S. Reker, and C. Urbach (ETM Collaboration), Lemon: an MPI parallel I/O library for data encapsulation using LIME, Comput. Phys. Commun. 183, 1321 (2012).

[56] A. Frommer, K. Kahl, S. Krieg, B. Leder, and M. Rottmann, Adaptive aggregation based domain decomposition multigrid for the lattice Wilson Dirac operator, SIAM J. Sci. Comput. 36, A1581 (2014).

[57] C. Alexandrou, S. Bacchio, J. Finkenrath, A. Frommer, K. Kahl, and M. Rottmann, Adaptive aggregation-based domain decomposition multigrid for twisted mass fermions, Phys. Rev. D 94, 114509 (2016).

[58] C. Alexandrou, S. Bacchio, and J. Finkenrath, Multigrid approach in shifted linear systems for the non-degenerated twisted mass operator, Comput. Phys. Commun. 236, 51 (2019).

[59] M. A. Clark, R. Babich, K. Barros, R. C. Brower, and C. Rebbi, Solving lattice QCD systems of equations using mixed precision solvers on GPUs, Comput. Phys. Commun. 181, 1517 (2010).

[60] R. Babich, M. A. Clark, B. Joo, G. Shi, R. C. Brower, and S. Gottlieb, Scaling Lattice QCD beyond 100 GPUs, in SC11 International Conference for High Performance Computing, Networking, Storage and Analysis Seattle, Washington (Association for Computing Machinery, New York, 2011) [arXiv:1109.2935].

[61] M. A. Clark, B. Joó, A. Strelchenko, M. Cheng, A. Gambhir, and R. Brower, Accelerating lattice QCD multigrid on GPUs using fine-grained parallelization, Proceedings of the International Conference for High Performance (IEEE Press, New York, 2016) [arXiv:1612.07873].

[62] R Development Core Team, R: A Language and Environment for Statistical Computing (R Foundation for Statistical Computing, Vienna, Austria, 2005). 